Journal of Case Reports 2018;8(1):51-53

\title{
One Lung Ventilation as a Cause of Obstructive Shock
}

\author{
J. Xu' ${ }^{1}$ M. D'souza1, K.V. Patel ${ }^{2}$, N. Naresh³ , T.D. Vagaonescu²
}

Departments of ${ }^{1}$ Internal Medicine, ${ }^{2}$ Cardiology, ${ }^{3}$ Pulmonary and Critical Care Medicine; Rutgers Robert Wood Johnson Medical School, New Brunswick, New Jersey.

\section{Corresponding Author: \\ Dr. Jack Xu \\ Email: Jaxu54@gmail.com}

This is an Open Access article distributed under the terms of the Creative Commons Attribution License (creativecommons.org/ licenses/by/3.0).

Received Accepted Published

September 25, 2017

February 1, 2018

February 15, 2018

\begin{abstract}
Background: Right heart failure (RHF) in the intensive care setting is an underdiagnosed, yet serious condition that carries a significant morbidity and mortality. The patho-physiology of RHF has been extensively studied and found to be most commonly caused by pulmonary embolism, acute cor-pulmonale, severe left ventricular failure, right ventricular infarction and in the setting sepsis. One rarely encountered etiology of RHF is one-lung-ventilation (OLV) leading to insurmountable right heart strain. Case Report: A 62 year old presented with recurrent urinary retention secondary to prostatic hypertrophy. Computed Tomography of chest showed mass in left upper lobe. Endo-bronchial biopsy of mass in left lung resulted in pulmonary hemorrhage. OLV via selective ventilation of only the right lung was employed which resulted in pulmonary hypertension and shock. Conclusion: We believe that in a hypotensive patient undergoing OLV, the practitioner must consider the possibility of obstructive shock secondary to the right heart strain generated by this technique. If RHF is suspected, we recommend obtaining an echocardiogram to detect signs of right heart strain. If strain in present, OLV must be stopped while the patient returns to hemodynamic stability. Further studies regarding the role of one lung ventilation as a cause of obstructive shock will be helpful.
\end{abstract}

Keywords: Heart Failure, Lung, One Lung Ventilation, Pulmonary Hypertension, Urinary Retention.

\section{Introduction}

Right heart failure (RHF) in the intensive care setting is an under-diagnosed, yet serious condition that carries a significant morbidity and mortality [1]. The patho-physiology of RHF has been extensively studied and found to be most commonly caused by pulmonary embolism, acute cor-pulmonale, severe left ventricular failure, right ventricular infarction and in the setting sepsis [1-6]. One rarely encountered etiology of RHF is one-lung-ventilation (OLV) leading to insurmountable right heart strain.

One-lung ventilation is a technique that can be used to collapse the non-ventilated lung to have better access and visualization of the thoracic cavity. The other major utility of OLV is to isolate a damaged lung. The goal in these scenarios is to either decrease the air pressure, in cases of fistulas or ruptured bullae, or to decrease blood flow, in cases of pulmonary hemorrhage. By decreasing either perfusion or ventilation to the diseased lung, the pathology is contained to the ipsilateral side and the contralateral lung is protected from cross contamination [2]. Here we describe a case of OLV leading to RHF and obstructive shock.

\section{Case Report}

A 62-year-old male with hepatitis $\mathrm{C}$, benign prostatic hypertrophy presented with recurrent urinary retention and was found to have an enlarged exophytic prostate mass. Computed tomography 
(CT) scan of the chest/abdomen/pelvis revealed a mass in the upper lobe of the left lung and a nodule in the upper lobe of the right lung. In order to further analyze the lung masses, an endo-bronchial biopsy was performed but the procedure was complicated by left-sided pulmonary hemorrhage.

The patient was immediately intubated in order to protect the airway and a trans-catheter artery embolization was attempted. The procedure failed to localize the lesion and the left sided hemorrhage continued to expand. In order to control the bleeding on the left side and prevent complications on the right, one-lung-ventilation (OLV) was employed via selective ventilation of only the right lung. Echocardiogram performed prior to OLV initiation was significant for an ejection fraction (EF) of $32 \%$, a severe decrease in left ventricular (LV) systolic function, mild tricuspid valve regurgitation, normal right ventricular (RV) cavity size and systolic function, and an RV systolic pressure (RVSP) of $39 \mathrm{mmHg}$.

Over the next several days, the patient's hemodynamic status steadily declined until he was found to be markedly hypotensive and in shock. It was initially thought that he was undergoing cardiogenic shock, given that his LV systolic function was decreased at the start of OLV and mixed venous oxygen saturation was 33\%. Flo Trac $\AA$ monitoring indicated normal cardiac output, so a follow-up echocardiogram was obtained and vasopressors were started. This study showed evidence of severe right heart strain and RHF; there was an increase in right atrial (RA) and RV cavity size, a decrease in RV systolic function, RVSP was $65 \mathrm{mmHg}$, and there was severe tricuspid valve regurgitation. Additionally, the EF was $25 \%$ and there was evidence of volume overload.

The inciting factor for the right heart strain and accompanying RHF was thought to be the pulmonary hypertension that resulted from OLV. Therefore ventilation was provided to both lungs and the patient's hemodynamic and respiratory status began to improve. He was then extubated and was successfully weaned off vasopressors. Follow-up echocardiogram showed an EF between $40-45 \%$ and a marked reduction in right heart strain.

\section{Discussion}

The right ventricle and the right side of the heart are developed to function in a low-pressure environment and are significantly compliant. This compliance allows the right heart to deal well with increases in volume, but not with increases in pressure [6]. While working against high pulmonary artery pressures, the right ventricle will hypertrophy which results in ischemic changes and dilation of the right heart [6]. RV dilatation can subsequently lead to clinically significant heart failure by two main mechanisms. Either the dilation results in decreased contractility and decreased RV systolic function or the dilation causes a displacement of the inter-ventricular septum into the LV, thereby increasing LV end-diastolic pressure and reduced LV systolic function [6].

Right heart failure (RHF) in the intensive care setting occurs most commonly due to infarction, acute pulmonary embolism, sepsisinduced hypovolemia, and pulmonary hypertension [5-7]. The way to confirm right heart failure at the bedside is through echocardiogram [5]. Echocardiogram findings that indicate right heart strain or failure include right atrial enlargement, tricuspid valve regurgitation, concentric shape of $\mathrm{RV}$, paradoxical septal movement in systole, and RV wall hypokinesis [4].

One-lung ventilation is not a common cause of right heart failure. OLV has been shown to have minimal changes in cardiopulmonary measurements (cardiac index, heart rate, mean arterial pressure, mean pulmonary artery pressures, pulmonary vascular resistance and $\mathrm{SvO}_{2}$ ) in supine patients [7]. However, it has been shown to increase right ventricular end diastolic area but has no effect 
on right ventricle fractional area change or tricuspid annular plane systolic excursion [7]. This increase in right ventricular end diastolic area is thought to be due to the increase in ventricular after-load from one-lung-ventilation being compensated by an increase in preload in order to maintain stroke volume [8].

In this case, it was thought that the right heart strain was secondary to one lung ventilation for total four day duration. Once two-lung ventilation was resumed, and then the patient was subsequently extubated, follow-up echocardiogram showed an increase of $15 \%$ in EF and improvement in right heart strain. As a result, he became more hemodynamically stable and eventually weaned off vasopressors.

\section{Conclusion}

In a hypotensive patient undergoing OLV, the practitioner must consider the possibility of obstructive shock secondary to the right heart strain generated by this technique. If RHF is suspected, we recommend obtaining an echocardiogram to detect signs of right heart strain. If strain in present, OLV must be stopped while the patient returns to hemodynamic stability. Further studies regarding the role of one lung ventilation as a cause of obstructive shock will be helpful.
Contributors: MD contributed in gathering information, writing introduction, case report and discussion. JX, MD, $\mathrm{KP}, \mathrm{NN}, \mathrm{TV}$ : manuscript editing, critical inputs and literature search. JX will act as guarantor. All authors approved the final version of this manuscript.

Funding: None; Competing interests: None stated.

\section{References}

1. Zochios V, Jones N. Acute right heart syndrome in the critically ill patient. Heart, Lung and Vessels. 2014;6:157-170.

2. Alaverdian A, Cohen R. The right ventricle in critical illness. The Open Critical Care Medicine Journal. 2010;3:38-42.

3. Chan C, Klinger J. The right ventricle in sepsis. Clinics in Chest Medicine, 2008;29:661-676.

4. Piazza G, Goldhaber SZ. The acutely decompensated right ventricle: pathways for diagnosis and management. Chest. 2005;128:1836-1852.

5. Lahm T, McCaslin CA, Wozniak TC, Ghumman W, Fadl $\mathrm{YY}$, Obeidat OS, et al. Medical and surgical treatment of acute right ventricular failure. J Am Coll Cardiol. 2010;56:1435-1446.

6. Markel T, Wairiuko G, Lahm T, Crisostomo P, Wang $\mathrm{M}$, Herring $\mathrm{C}$, et al. The right heart and its distinct mechanisms of development, function, and failure. Journal of Surgical Research. 2008;146:304-313.

7. Kashtan H, Brown D. Cardiopulmonary effects of one-lung ventilation in supine patients. Journal of Cardiothoracic Anesthesia. 1988;2:643-645.

8. Wilkinson JN, Scanlan M, Skinner H, Malik M. Right heart function during one-lung ventilation-observations using transoesophageal echocardiography. Anaesthesia. 2009;64:1387-1389. 